\title{
Effects of a self-management program on antiemetic-induced constipation during chemotherapy among breast cancer patients: a randomized controlled clinical trial
}

\author{
Akiko Hanai $^{1} \cdot$ Hiroshi Ishiguro $^{1} \cdot$ Takashi Sozu $^{2} \cdot$ Moe Tsuda $^{1} \cdot$ Hidenori Arai $^{3}$. \\ Akira Mitani $^{1} \cdot$ Tadao Tsuboyama $^{1}$
}

Received: 24 November 2015/ Accepted: 26 November 2015/Published online: 9 December 2015

(c) The Author(s) 2015. This article is published with open access at Springerlink.com

\begin{abstract}
Research on patient-reported outcomes indicates that constipation is a common adverse effect of chemotherapy, and the use of 5-hydroxytryptamine (serotonin; $5 \mathrm{HT}_{3}$ ) receptor antagonists aggravates this condition. As cancer patients take multiple drugs as a part of their clinical management, a non-pharmacological self-management (SM) of constipation would be recommended. We aimed to evaluate the effectiveness of a SM program on antiemetic-induced constipation in cancer patients. Thirty patients with breast cancer, receiving $5 \mathrm{HT}_{3}$ receptor antagonists to prevent emesis during chemotherapy were randomly assigned to the intervention or control group. The SM program consisted of abdominal massage, abdominal muscle stretching, and education on proper defecation position. The intervention group started the program before the first chemotherapy cycle, whereas patients in the wait-list control group received the program on the day before their second chemotherapy cycle. The primary outcome was constipation severity, assessed by the constipation assessment scale (CAS, sum of eight components). The secondary outcome included each CAS component (0-2 points) and mood states. A self-reported assessment of satisfaction with the program was performed. The program produced a statistically and clinically significant alleviation of constipation severity (mean difference in CAS,
\end{abstract}

Electronic supplementary material The online version of this article (doi:10.1007/s10549-015-3652-4) contains supplementary material, which is available to authorized users.

\footnotetext{
Hiroshi Ishiguro

hkkishi@kuhp.kyoto-u.ac.jp

1 Graduate School of Medicine, Kyoto University, 54 Shogoinkawaharacho, Sakyo-ku, Kyoto 606-8507, Japan

2 Tokyo University of Science, Tokyo, Japan

3 National Center for Geriatrics and Gerontology, Aichi, Japan
}

$-3.00 ; P=0.02)$, decrease in the likelihood of a small volume of stool $(P=0.03)$, and decrease in depression and dejection $(P=0.02)$. With regards to program satisfaction, 43.6 and $26.4 \%$ patients rated the program as excellent and good, respectively. Our SM program is effective for mitigating the symptoms of antiemetic-induced constipation during chemotherapy.

Keywords Constipation - Antiemetic - Breast cancer . Supportive care $\cdot$ Exercise $\cdot$ Self-management

\section{Introduction}

Research on patient-reported outcomes in cancer treatment indicates that constipation is one of the most common and distressing side effects of cancer therapy [1,2]. The use of 5-hydroxytryptamine (serotonin; $5 \mathrm{HT}_{3}$ ) receptor antagonist, which is commonly included as part of the pharmacologic treatment of cancer to alleviate emetogenesis, often increases the severity of constipation [3-5]. Although constipation is a significant health issue related to chemotherapy, the incidences of constipation (defined by the Common Terminology Criteria for Adverse Events version 4.0 or CTCAEv4.0 for short) in clinical trials are frequently underreported as physicians are often not made aware of this symptom [4]. The incidence of $5 \mathrm{HT}_{3}$ receptor antagonistinduced constipation increases in conjunction with the emetogenic risk of the chemotherapy medication used. For instance, the incidence rate of constipation with the use of 5-fluorouracil-epidoxorubicin-cyclophosphamide, a regimen with a high emetic risk, is reported to be as high as $84 \%$, compared to a constipation incidence of $50 \%$ with use of low emetic risk drugs, such as docetaxel and paclitaxel $[4,5]$. The combination of a $5 \mathrm{HT}_{3}$ receptor antagonist 
and a corticosteroid is recommended as an antiemetic prophylaxis in patients with a high or moderate-to-high emetic risk, whereas $5 \mathrm{HT}_{3}$ is not routinely prescribed to patients with a low emetic risk [6].

Physical exercise facilitates bowel movement and is recommended for patients with chronic constipation or irritable bowel syndrome [7-12]. Abdominal massage has also been emphasized as a non-pharmacological intervention to improve chronic constipation [13-15]. Additionally, a proper defecation position can ensure the smooth passage of stool during defecation [16-18]. As cancer patients often take multiple medications to alleviate the side effects of chemotherapy, a non-pharmacological intervention to alleviate constipation would be favorable, while avoiding the adverse side effects commonly associated with laxatives, including bloating, increased gas, and abdominal fullness.

Self-care management and lifestyle education during cancer treatment are important to minimize antiemetic-induced constipation [3, 19]. Therefore, the aim of our clinical trial was to evaluate the effectiveness of a selfmanagement (SM) program in mitigating the symptoms of antiemetic-induced constipation in cancer patients.

\section{Patients and methods}

\section{Study design and patients}

A randomized, waiting-list controlled, parallel group, open-label pilot trial was conducted. This trial was approved by the ethics committee of Kyoto University Graduate School and Faculty of Medicine, and was registered with the University Hospital Medical Information Network in February 2013 (UMIN000009676).

Breast cancer patients were recruited from Kyoto University Hospital, Kyoto, Japan, between February 2013 and March 2014, according to the following inclusion criteria: suitable candidate for chemotherapy; planned prescription of $5 \mathrm{HT}_{3}$ receptor antagonist, including ondansetron, granisetron, tropisetron, or palonosetron; an Eastern Cooperative Oncology Group Performance Status of 0 or 1 ; ability to defecate and normal digestive and abdominal functions before study enrollment; and provision of signed informed consent. Prospective patients were further screened on the following exclusion criteria: difficulty communicating due to a mental disorder, cognitive impairment, or physical disability; constipation severity score higher than a CTCAEv4.0 grade 2 before enrollment; pregnancy; restrictions or contraindications to exercise; not suitable for study inclusion based on the primary physician's judgment; and indication of intent to continue taking non-prescribed supplements. Note that the final exclusion criterion was added to the protocol in July 2013 and this change did not affect the measured outcome of the previously enrolled patients as the use of non-prescribed supplements has consistently been strongly discouraged during chemotherapy because of possible drug-drug interactions.

Independent research staff performed block randomization of recruited patients into the intervention or the control group, using a block size of 4 and a computergenerated random-numbers table. Patients were randomized (1:1) into an intervention or waiting-list control group. An occupational therapist verified the allocation by phone and consulted with the patients in the intervention group regarding the SM program.

Patient-reported outcomes were assessed before chemotherapy (baseline), after the first cycle of chemotherapy (C1) in the hospital and after the second cycle of chemotherapy $(\mathrm{C} 2)$ through out-patient care. Activity monitors were provided only during $\mathrm{C} 1$.

\section{Standard care (provided to all patients)}

On the day before chemotherapy, general self-care information was provided for constipation regarding the use of medication (magnesium oxide, sennoside, and sodium carbonate suppository), and the benefits of food and drink intake, circadian rhythms, a relaxing environment, a bidet, and moderate exercise.

\section{Intervention}

The intervention group was educated on the SM program, which included (a) abdominal massage, (b) abdominal exercise, and (c) information on the proper defecation position, on the day before $\mathrm{C} 1$ (Fig. S1). This simple SM program was taught using an illustrated guide that patients could easily learn in approximately $10 \mathrm{~min}$. The waitinglist control group was educated on the program on the first day of $\mathrm{C} 2$, before chemotherapy was administered.

\section{Abdominal massage}

Patients were informed that abdominal massage could promote bowel motility throughout the colon (Fig. S1a) [15]. This massage was performed by applying a constant moderate pressure to the abdomen using two or three fingers. Small, clockwise circular movements were performed over the colon. Each pass of the massage required approximately $1 \mathrm{~min}$ and patients were instructed to repeat the massage 10 times per day.

\section{Abdominal exercise}

Three abdominal exercises (Fig. S1b, i-iii) and abdominal breathing were taught to patients. Based on a number of 
yoga poses and a cyclic abdominal stretching protocol for irritable bowel syndrome [12], these exercises were designed for breast cancer patients to stimulate and increase the movement of bowel content, while avoiding excessive compression or extension of the skin around the breasts. The three exercises are described below.

1. Wind-relieving Pose: Patients wrapped their hands around one knee and pulled it gently toward their chest and drew their head to their knees. They held the position for 15-30 s while taking slow, deep, calm breaths. The position, with end-position static hold, was repeated with the opposite knee.

2. Knees-to-chest Pose: Patients lay down on their back, drawing their knees toward their chest and interlacing their hands on top of their knees.

3. Reclined Spinal Twist: Patients lay down on their back with their knee flexed to approximately $90^{\circ}$ and their feet resting on a bed. Then, the trunk and pelvis were rotated and held at $80 \%$ of the patient's maximal rotation angle for $1 \mathrm{~min}$. The twisting position was then repeated using the other knee.

Following these exercises, patients performed abdominal breathing by lying on their back, placing their hands on their abdomen, and breathing deeply. These exercises required 5-6 min to complete, and patients were instructed to repeat them 10 times per day. Abdominal massage and abdominal exercise were logged by using an Actiwatch $^{\circledR}$ (Cambridge Neurotechnology, Ltd.) and by self-reporting by patients.

\section{Proper defecation position}

Defecating in a semi-squatting position [14, 15] was introduced to patients through an explanation of anorectal anatomy and abdominal and thoracic pressures during defecation (Fig. S1c). To maintain the proper position during excretion, a small step and cushion were provided.

\section{Outcome measures}

The primary endpoint was the constipation status, assessed using the constipation assessment scale (CAS). Secondary endpoints were each component of the CAS, mood states measured by the profile of mood states (POMS), and health-related quality of life (HRQOL). The frequency of laxative use and defecation, food and water intake, and rest-activity patterns, as well as adherence and satisfaction with the program were also measured. Outcomes were assessed before chemotherapy (baseline), at $\mathrm{C} 1$, and at $\mathrm{C} 2$.

The CAS is an eight-item, self-reported measure designed to assess the presence and severity of constipation [20-22]. The eight items evaluated by the CAS are abdominal distention or bloating; change in amount of gas passed rectally; less frequent bowel movements; rectal fullness or pressure; rectal pain with bowel movements; small volume of stool; inability to pass stool; and oozing liquid stool. The Japanese version of the CAS, which has been modified and validated to assess constipation in Japanese populations [21], was used. A low score on the CAS is indicative of less severe constipation.

The POMS is a self-administered test that identifies and assesses transient, fluctuating affective states in individuals [23-25]. The test assesses the following six components of mood, with higher scores indicative of greater disturbances: tension-anxiety, anger-hostility, fatigue-inertia, depression-dejection, vigor-activity, and confusion-bewilderment. In this study, the short version of the POMS was used.

The Short Form 36 Health Survey (SF36) assesses the following eight areas of general health: disease-related limitations in physical activities; limitations in social functioning as a result of physical and/or emotional problems; limitations in participating in a usual role or function (work or other daily activities) as a result of emotional problems; limitations in participating in a usual role or functioning as a result of physical health problems; bodily pain; general mental health (feelings about well-being or depression); vitality (energy and fatigue); and general health perceptions [26-28]. Scores ranging from 0 to 100 were calculated for each of these different health aspects, with a low score indicative of a poor health status. In this study, the standard version of the SF36 was used for baseline assessment, and the abbreviated version was used at the follow-up time points.

The wrist actigraph, Actiwatch ${ }^{\circledR}$, which is designed to provide accurate and objective sleep and wake activity, was used to determine abnormal rest-activity patterns [29, 30]. By clicking on the markers, a complete list of the times when the marker button on the side of the Actiwatch ${ }^{\circledR}$ has been pressed is provided. Patients wore the Actiwatch ${ }^{\circledR}$ continuously on their non-dominant forearm, and they pushed the marker when they slept and woke up to $\log$ their subjective rest-activity patterns. Patients in the intervention group were also required to push the marker when they started and finished the SM program to assess adherence objectively.

Laxative use, food and water intake, and frequency of defecation, nausea, or vomiting were also self-reported by patients. The daily use of laxatives (magnesium oxide, sennoside, or sodium carbonate suppository); amount of food and water intake; and frequency of defecation, nausea, or vomiting (CTCAEv 4.0) were reported throughout the trial.

The self-report satisfaction questionnaire included (1) ease of use; (2) perception of whether it was effective; (3) 
desire to continue; and (4) timing of the intervention. Patients were asked to respond to each question with a grade of "bad," "poor," "fair," "good," or "excellent." A free writing space was also provided to obtain subjective feedback. This questionnaire was added to the protocol in July 2013.

\section{Sample size and data analysis}

All the data were double checked by a third-party beneficiary. The data plausibility and the analysis were supervised by a statistician. Statistical analyses were performed using the full analysis set (Fig. 1). Age, weight, and body mass index (BMI) are presented as mean and standard deviation (SD). The regimen of chemotherapy and dose of palonosetron are presented according to the number of patients. The total CAS score-a primary endpoint after $\mathrm{C} 1$-was adjusted by the baseline score (C1-baseline), is presented as mean (SD), and was compared between the two groups using Student's $t$ test. Each component of the CAS was adjusted by the baseline score (C1-baseline), is presented as mean (SD), and was analyzed using the Wilcoxon rank-sum test. To perform a graphical evaluation of each CAS component, radar plots were created to report the dimension scores. Mood states and the HRQOL are presented as mean (SD), and were analyzed using the Wilcoxon rank-sum test.

The number of patients who showed abnormal rest-activity patterns, as assessed by actigraphy, and nausea and vomiting, as assessed by the CTCAEv 4.0 grade 2, was also determined. The use of laxatives, food intake, and frequency of defecation are presented as mean (SD). The patients' impression and satisfaction with the program are presented using percentage bar charts, based on the number of patients for each answer.

The sample size was not determined based on a formal power calculation but rather on the number of patients that could realistically be recruited from the target patient

Fig. 1 CONSORT diagram

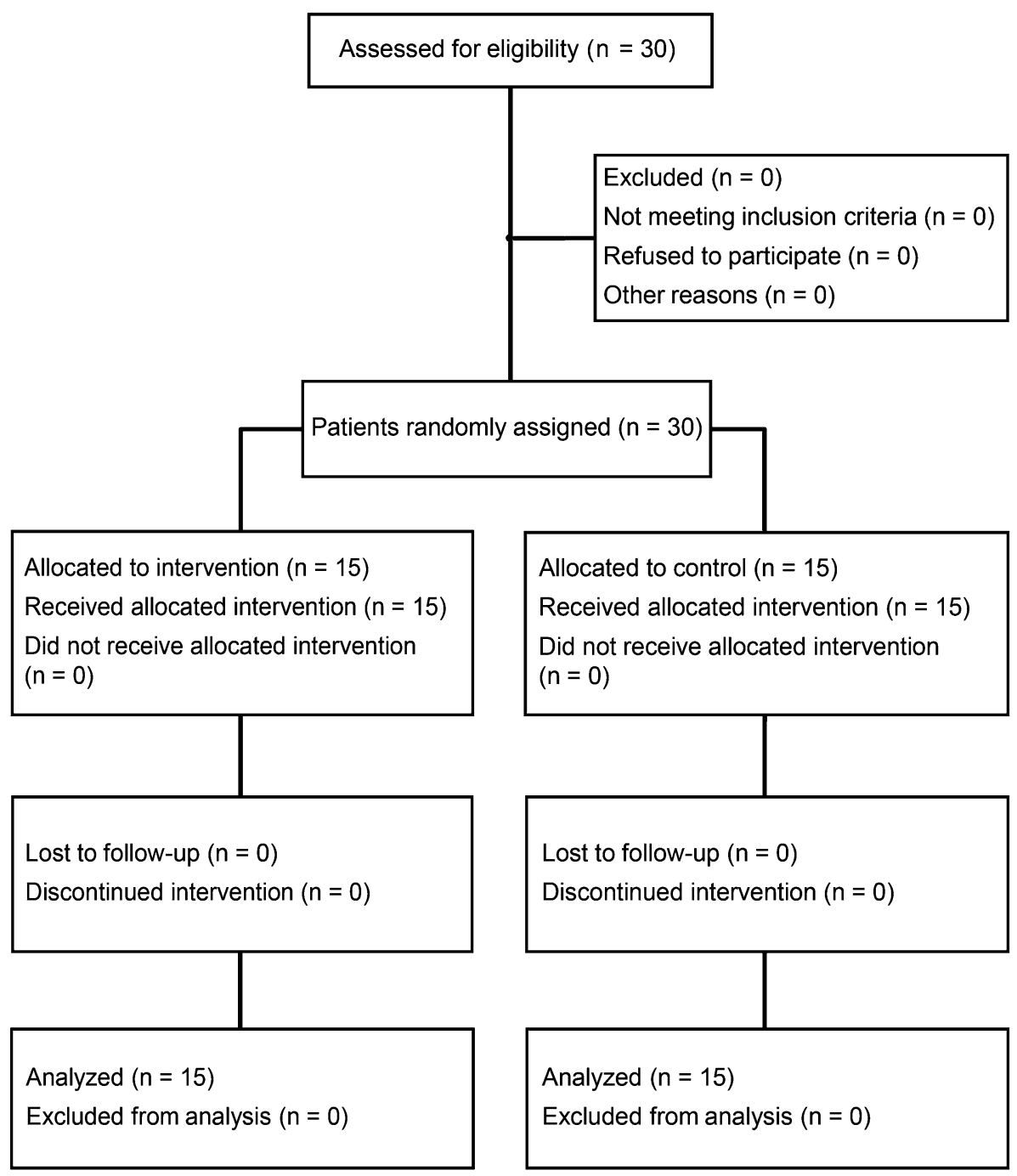


population within the study period. With 30 patients enrolled in the study, our sample size was similar to a previous trial [13].

All $P$ values were two-sided, and $P$ values $<0.05$ were considered statistically significant. All statistical analyses were performed using SAS, version 9.3 (SAS Institute Inc.) and $\mathrm{R}$ (R Foundation for Statistical Computing).

\section{Results}

\section{Patient recruitment and characteristics}

Thirty patients who met the eligibility criteria were randomized (1:1) into an intervention or waiting-list control group. Table 1 summarizes patients' characteristics. Age, weight, BMI, and chemotherapy regimen were comparable between the intervention and control groups.

\section{Adherence to the intervention program}

All patients received the same instructions regarding the goals of the program and the manner in which it can be performed. Patients in the intervention group performed abdominal massage, on average, 3.82 times per day and abdominal muscle stretching 3.57 times per day. No patients in either group reported exercise-induced stomach discomfort, and all preferred continuing the SM program during chemotherapy.

\section{Primary endpoint}

Figure 2 shows the change in antiemetic-induced constipation during chemotherapy as a proportional change of the total CAS score. The change in the CAS score was adjusted by the baseline score and plotted using a boxplot. The mean change in the CAS was significantly and clinically lower in the intervention group, compared to the control, with a mean between-group difference of approximately $40 \%$ [mean difference $=-3.00 ; 95 \%$ confidence interval (CI), -5.46 to $-0.54 ; P=0.019]$.

All the patients in the waiting-list control group were educated regarding the program after the $\mathrm{C} 1$ (no intervention) assessment and just prior to $\mathrm{C} 2$. In Fig. 2, the box on the right shows the score after $\mathrm{C} 2$, adjusted by the baseline score, for the waiting-list control group. Patients after the intervention program (C2) reported a lower severity of constipation as compared to the period when they had were yet instructed for the intervention program, the boxplot in the middle (C1). Changes for each patient in the intervention and control groups (baseline, C1, and C2) are shown in Fig. 3.

\section{Secondary endpoints}

Figure 4 shows the individual scores of the eight components of the CAS on a radar plot. There was a significant between-group difference in the component of small volume of stool $(P=0.032)$. The score of each CAS component at $\mathrm{C} 1$ and $\mathrm{C} 2$ is shown in Table $\mathrm{S} 1$.
Table 1 Relevant characteristics of the study group

\begin{tabular}{lccc}
\hline & Intervention $(n=15)$ & Control $(n=15)$ & Overall $(n=30)$ \\
\hline $\begin{array}{l}\text { Demographic profile, mean (SD) } \\
\text { Age (years) }\end{array}$ & $55.3(12.6)$ & $54.9(9.7)$ & $55.1(11.0)$ \\
$\begin{array}{l}\text { Medical profile, mean (SD) } \\
\text { Weight }(\mathrm{kg})\end{array}$ & $54.8(9.6)$ & $56.7(10.0)$ & $55.8(9.7)$ \\
BMI $\left(\mathrm{kg} / \mathrm{m}^{2}\right)$ & $22.4(4.3)$ & $23.0(4.2)$ & $22.7(4.2)$ \\
Chemotherapy, $\mathrm{n}(\%)$ & & & $18(60.0)$ \\
High emetic risk & $8(53.3)$ & $5(33.3)$ & $12(40.0)$ \\
Moderate emetic risk & $7(46.7)$ & $4(26.7)$ & $7(23.3)$ \\
Taxotere or docetaxel use & $3(20.0)$ & & $27(90.0)$ \\
Palonosetoron use, $\mathrm{n}(\%)$ & & $14(93.3)$ & $3(10.0)$ \\
$0.75 \mathrm{mg}$ & $13(86.7)$ & $1(6.7)$ & \\
$0.30 \mathrm{mg}$ & $2(13.3)$ & & \\
\hline
\end{tabular}

$S D$ standard deviation 


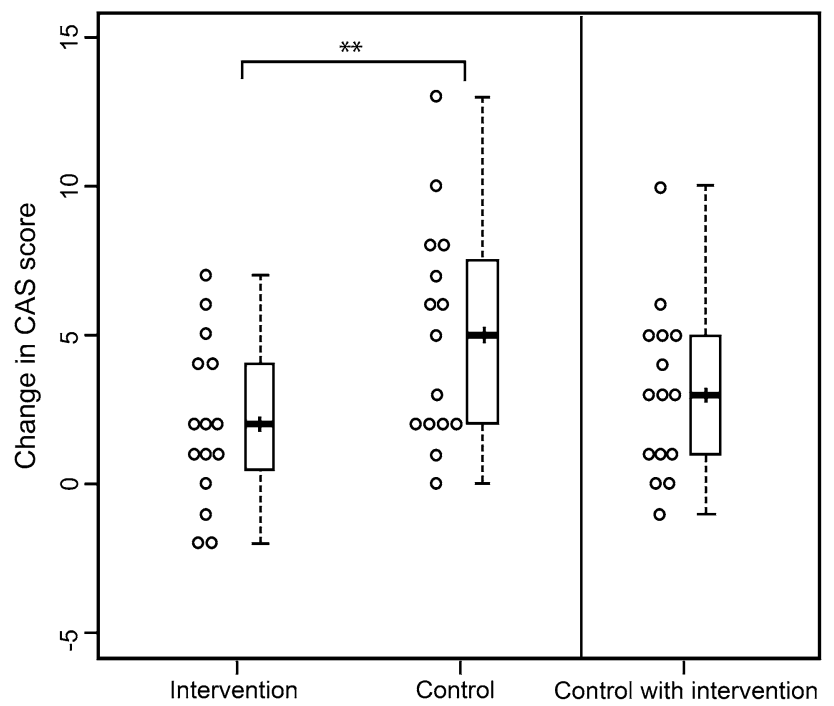

Fig. 2 Change in findings of self-evaluation of antiemetic-induced constipation. The 2 boxes on the left indicate the score after the first cycle of chemotherapy ( $\mathrm{C} 1$ ), as adjusted by the baseline score (C1-baseline). The mean change in the constipation assessment scale (CAS) was significantly lower in the intervention group than in the control group [mean, 2.0 vs. $5.0 ; 95 \%$ confidence interval $(-5.46$ to $-0.54) ;{ }^{*} P=0.019, t$ test]. The box on the right indicates the score after the second cycle of chemotherapy (C2), as adjusted by the baseline score (C2-baseline) in the control group (waiting-list control $\mathrm{C} 1$ without intervention in the hospital; $\mathrm{C} 2$ with intervention via outpatient care)

As evident in Table S2, the depression-dejection score of the POMS was significantly lower for the intervention group, compared to the control group $(P=0.021)$. No between-group differences were identified for tensionanxiety, anger-hostility, vigor, fatigue, or confusion.

The intervention had no significant effect on HRQOL (Table S2). No notable differences were observed in the use of laxatives, food intake, and frequency of defecation, nausea, or vomiting between the groups (Table S3). No patients showed abnormal rest-activity patterns, such as reverse cycling or sleeplessness.

In the satisfaction questionnaire, nearly half of the patients $(43.6 \%)$ rated the program as excellent, whereas $26.4 \%$ rated it as good (Fig. 5).

\section{Discussion}

Our study findings support our priori hypothesis that a SM program could mitigate the symptoms of antiemetic-induced constipation. The severity of constipation was approximately $40 \%$ lower in the intervention group, compared to the control group, with a mean CAS score of $<5$ points in the intervention group, which is indicative of mild constipation not requiring medical intervention. With regard to the mood states, depression-dejection was less
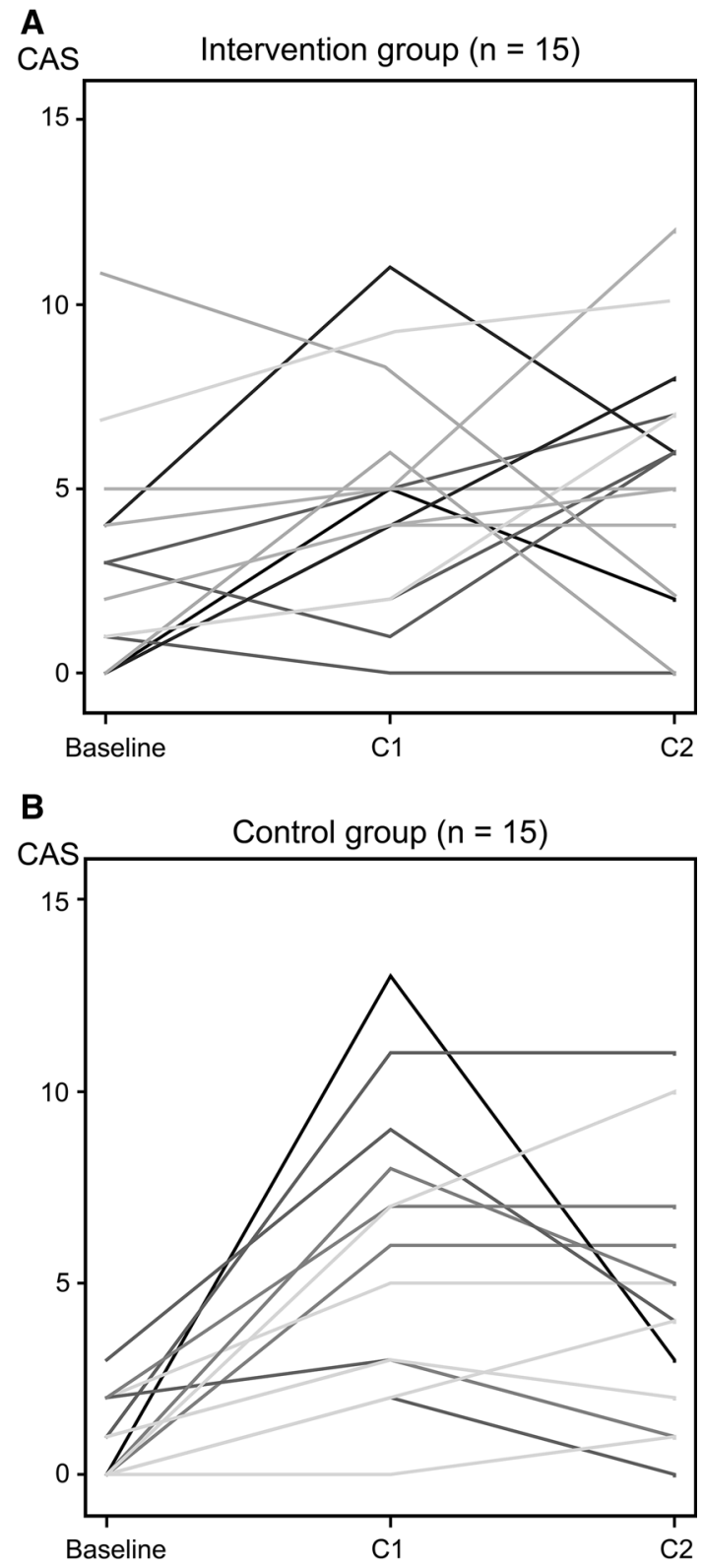

Fig. 3 Change in the constipation assessment scale (CAS) results

frequently reported by the intervention group than by the control group. Over half of the patients in both groups were satisfied with the program and preferred to continue the program during chemotherapy. These findings indicate the clinical applicability of the program; moreover, the intervention does not require costly resources and is not labor extensive.

The limitations of our study should be noted in interpreting outcomes of our trial and clinical application. First, only short-term effects of the SM program on constipation were evaluated (i.e., 4 days of the first cycle of chemotherapy). Although a longitudinal analysis was not performed, the duration of assessment used in our trial was 
Fig. 4 Radar chart for the components of the constipation assessment scale, adjusted by the baseline score, with $P$ values determined by the Wilcoxon rank-sum test. The solid line shows the score for the intervention group and the dotted line shows the score for the control group

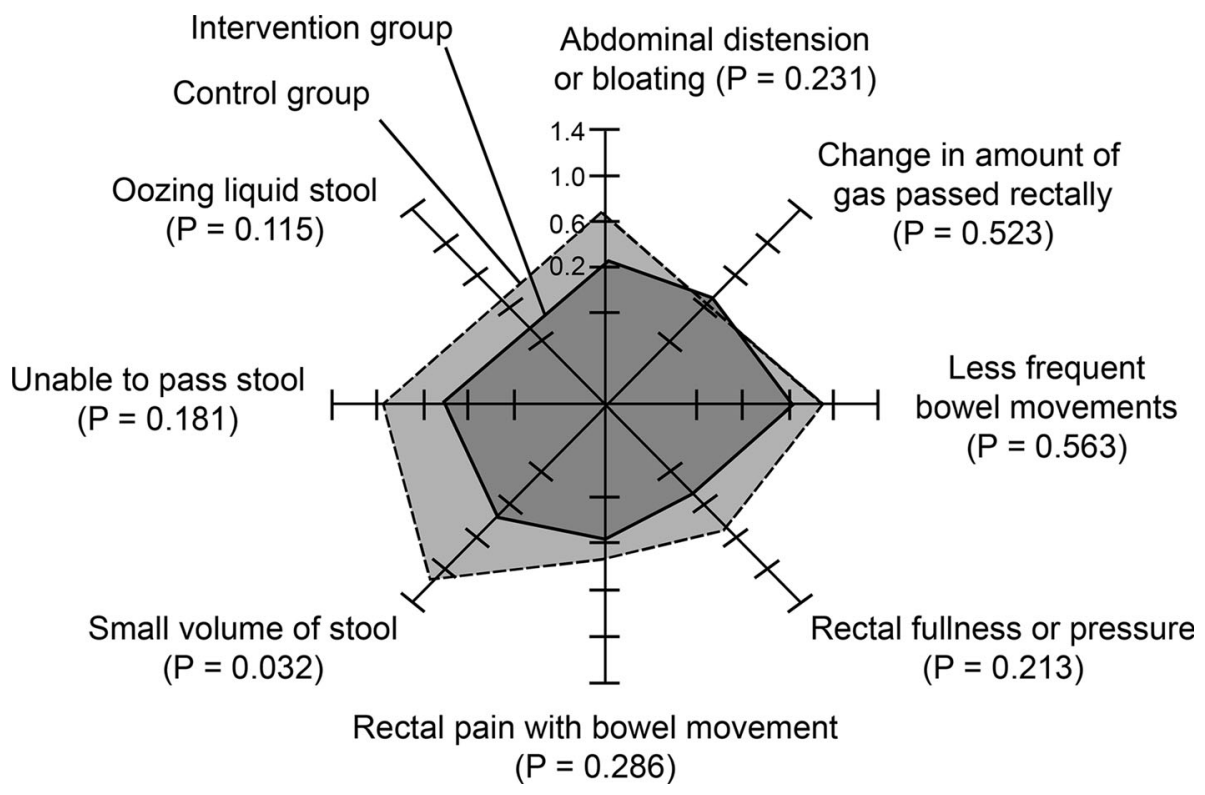

quality of life, compared to CTCAE [31]. Fourth, the mean difference in CAS score between the intervention and control groups was relatively small. As the CAS uses a checklist for constipation symptoms, the overall scores tend to be lower; for instance, the score for morphineinduced severe constipation was 7.6/16. In general, 5 points on the CAS is indicative of severe constipation requiring medical intervention, compared to $0-2$ points indicating minor symptoms not requiring intervention [20, 21]. Hence, even a small difference (5 points on the CAS in the control group vs. 2 points on the CAS in the intervention group) would be significant in clinical situations.

A recent review reported that exercises or massage are effective to alleviate other types of constipation, although there was previously no evidence that a SM program could help mitigate $5 \mathrm{HT}_{3}$ receptor antagonist-induced constipation [8, 11, 13-15]. Bidirectional brain-gut interactions involving 5-HT pathways are important in gastrointestinal functioning [32-35]. Although psychological well-being may cause additional expectancy effects in patient-reported outcomes, it is difficult to fractionate constipation and psychological states [32-35]. According to a previous study, increases in physical activity or SM skills may stimulate physical functioning, such as the volume of stool, reduce the occurrence of severe depressive symptoms, and promote emotional well-being [32, 36, 37]. This simple, effective, comfortable, and low-cost program for antiemetic-induced constipation may apply to other types of cancer patients or patients who suffer from other types of constipation, such as opioid-induced or chronic constipation.

In conclusion, for breast cancer patients who undergo chemotherapy and control nausea and vomiting using outcomes were used due to their higher concordance with 
$5 \mathrm{HT}_{3}$ receptor antagonists, our program offers a precise method for managing abdominal discomfort. Future trials, with a long-term follow-up, a wider population range, and placebo-controlled design are needed to verify the longitudinal and general effect of this SM program.

Acknowledgments We gratefully acknowledge the help provided by Prof. T. Futaki, Prof. M. Toichi, Prof. T. Hamaguchi, and the medical staff (physicians, nurses, and pharmacists) of Kyoto University Hospital. This material is based on work supported by the Japan Society for the Promotion of Science and by the Promotion Plan for the Platform of Human Resource Development for Cancer administered by the Ministry of Education, Culture, Sports, Science and Technology in Japan.

\section{Compliance with ethical standards}

Conflicts of interest The authors indicate no potential conflicts of interest.

Open Access This article is distributed under the terms of the Creative Commons Attribution-NonCommercial 4.0 International License (http://creativecommons.org/licenses/by-nc/4.0/), which permits any noncommercial use, distribution, and reproduction in any medium, provided you give appropriate credit to the original author(s) and the source, provide a link to the Creative Commons license, and indicate if changes were made.

\section{References}

1. U.S. Department of Health and Human Services: common terminology criteria for adverse events (CTCAE) version 4.0. http:// www.jcog.jp/doctor/tool/CTCAEv4J_20111217.pdf

2. Gibson RJ, Keefe DM (2006) Cancer chemotherapy-induced diarrhoea and constipation: mechanisms of damage and prevention strategies. Supp Care Cancer 14:890-900

3. Eisenberg P, Figueroa-Vadillo J, Zamora R et al (2003) Improved prevention of moderately emetogenic chemotherapy-induced nausea and vomiting with palonosetron, a pharmacologically novel 5- $\mathrm{HT}_{3}$ receptor antagonist. Cancer 98:2473-2482

4. Kimura M, Usami E, Yasuda T et al (2007) Analysis of adverse reactions in cancer chemotherapy: constipation occurring with co-administration of anticancer agents and 5-HT_3 antagonists. JSPHCS 33:863-868

5. Takei A, Fukuda Y, Seyama R et al (2008) Nursing support based on the characteristics of adverse reactions to outpatient chemotherapy: an analysis of adverse reactions recorded in patients' diaries. Ann Gunma Univ Sch Health Sci 29:11-20

6. Gralla RJ, Osoba D, Kris MG et al (1999) Recommendations for the use of antiemetics: evidence-based, clinical practice guidelines. J Clin Oncol 17:2971-2994

7. Courneya KS, Segal RJ, Mackey JR et al (2007) Effects of aerobic and resistance exercise in breast cancer patients receiving adjuvant chemotherapy: a multicenter randomized controlled trial. J Clin Oncol 25:4396-4404

8. Bi L, Triadafilopoulos G (2003) Exercise and gastrointestinal function and disease: an evidence-based review of risks and benefits. Clin Gastroenterol Hepatol 1:345-355

9. Jim HS, Small B, Faul LA et al (2011) Fatigue, depression, sleep, and activity during chemotherapy: daily and intraday variation and relationships among symptom changes. Ann Behav Med 42:321-333

10. McMillan SC (2004) Assessing and managing opiate-induced constipation in adults with cancer. Cancer Control 11:3-9

11. Vasanwala FF (2009) Management of chronic constipation in the elderly. SFP 35:84-92

12. Hamaguchi T, Fukudo S, Kanazawa M et al (2008) Changes in salivary physiological stress markers induced by muscle stretching in patients with irritable bowel syndrome. Biopsychosoc Med 2:20

13. Lämås K, Lindholm L, Stenlund $\mathrm{H}$ et al (2009) Effects of abdominal massage in management of constipation-A randomized controlled trial. Int J Nurs Stud 46:759-767

14. Lai T, Cheung M, Lo C et al (2011) Effectiveness of aroma massage on advanced cancer patients with constipation: a pilot study. Complement Ther Clin Pract 17:37-43

15. Harrington KL, Haskvitz EM (2006) Managing a patient's constipation with physical therapy. Phys Ther 86:1511-1519

16. Ernst E (1999) Abdominal massage therapy for chronic constipation: a systematic review of controlled clinical trials. Forsch Komplementarmed 6:149-151

17. Vasanwala FF (2009) Management of chronic constipation in the elderly. SFP 35:84-92

18. Sikirov D (2003) Comparison of straining during defecation in three positions: results and implications for human health. Dig Dis Sci 48:1201-1205

19. McMillan SC, Williams FA (1989) Validity and reliability of the constipation assessment scale. Cancer Nurs 12:183-188

20. Fukai K, Sugita A, Tanaka M (1995) A developmental study of the Japanese version of the constipation assessment scale. JJNS 28:201-207

21. Nagaviroj K, Yong WC, Fassbender K et al (2011) Comparison of the constipation assessment scale and plain abdominal radiography in the assessment of constipation in advanced cancer patients. J Pain Symptom Manage 42:222-228

22. Curran SL, Andrykowski MA, Studts JL et al (1995) Short form of the profile of mood states (POMS-SF): psychometric information. Psychol Assessment 7:80-83

23. Yokoyama K, Araki S, Kawakami N et al (1990) Production of the Japanese edition of profile of mood states (POMS): assessment of reliability and validity. Nihon Koshu Eisei Zasshi 37:913-918

24. Yokoyama K, Araki S (2002) A manual of POMS Japanese version. Kaneko, Tokyo

25. Ware JE Jr, Sherbourne CD (1992) The MOS 36-item short-form health survey (SF-36): I. Conceptual framework and item selection. Med Care 30:473-483

26. Fukuhara S, Bito S, Green J et al (1998) Translation, adaptation, and validation of the SF-36 health survey for use in Japan. J Clin Epidemiol 51:1037-1044

27. Fukuhara S, Ware JE Jr, Kosinski M et al (1998) Psychometric and clinical tests of validity of the Japanese SF-36 health survey. $\mathrm{J}$ Clin Epidemiol 51:1045-1053

28. Neurotechnology C (1999) The actiwatch activity monitoring system. Instructions for Use and Software Manual, Cambridge, UK

29. Gironda RJ, Lloyd J, Clark ME et al (2007) Preliminary evaluation of reliability and criterion validity of Actiwatch-Score. J Rehabil Res Dev 44:223-230

30. Basch E, Jia X, Heller G et al (2009) Adverse symptom event reporting by patients vs clinicians: relationships with clinical outcomes. J Natl Cancer Inst 101:1624-1632

31. Müller-Lissner SA, Kamm MA, Scarpignato C et al (2005) Myths and misconceptions about chronic constipation. Am J Gastroenterol 100:232-242 
32. Rhee SH, Pothoulakis C, Mayer EA (2009) Principles and clinical implications of the brain-gut-enteric microbiota axis. Nat Rev Gastroenterol Hepatol 6:306-314

33. Dunn WB, Broadhurst DI, Atherton HJ et al (2011) Systems level studies of mammalian metabolomes: the roles of mass spectrometry and nuclear magnetic resonance spectroscopy. Chem Soc Rev 40:387-426

34. Crowell MD (2004) Role of serotonin in the pathophysiology of the irritable bowel syndrome. Br J Pharmacol 141:1285-1293

35. Cheng C, Chan AOO, Hui WM et al (2003) Coping strategies, illness perception, anxiety and depression of patients with idiopathic constipation: a population-based study. Aliment Pharmacol Ther 18:319-326

36. Bodenheimer T, Lorig K, Holman H et al (2002) Patient selfmanagement of chronic disease in primary care. JAMA 288:2469-2475

37. Penedo FJ, Dahn JR (2005) Exercise and well-being: a review of mental and physical health benefits associated with physical activity. Curr Opin Psychiatry 18:189-193 